\title{
Representaciones de docentes universitarios sobre la deserción de los estudiantes de primer año en modalidad semipresencial pertenecientes a la Universidad UNIACC
}

doi: 10.33264/rpa.201802-09

Cristian Ortega Bustos, Anais Aluicio González, Escuela de Psicología, Edith Jorquera Muñoz, Escuela de Humanidades Facultad de Humanidades y Ciencias Sociales, UNIACC

\section{Resumen}

La presente investigación tuvo por objetivo explorar las representaciones que tienen docentes universitarios de la universidad UNIACC respecto de la deserción de los estudiantes de primer año que cursan estudios en modalidad semipresencial. Se realizó un estudio cualitativo de corte transversal con alcance exploratorio a través de cuatro entrevistas. Para el análisis de los datos se utilizaron los procedimientos propuestos por la Grounded Theory. Los resultados muestran cuatro categorías descriptivas y dos categorías relaciones que permiten aproximarse a las representaciones que tienen los docentes universitarios respecto a la deserción universitaria. Se discute acerca de la desmotivación y la frustración que pueden llegar a experimentar los estudiantes como elementos comprensivos del fenómeno y se proponen reflexiones para abordarla.

Palabras clave: b-learning, deserción universitaria, persistencia académica, educación virtual, educación semipresencial.

\section{Abstract}

The aim of this research was to explore the representations of university professors from the UNIACC University regarding the phenomenon of the desertion of first-year students who study b-learning. A qualitative cross-sectional study with exploratory scope was conducted, with a total of four interviews. The analysis of the data was performed according to the procedures proposed by the Grounded Theory. The results show four descriptive categories and two categories that permit to approximate the representations that the university teachers have regarding the university dropout. There is a discussion about the demotivation and frustration that students can experience as comprehensive elements of the phenomenon, and reflections are proposed to address it.

Keywords: b-learning, university desertion, academic persistence, virtual education, blended education. 


\section{Introducción}

La educación superior ha experimentado cambios sustantivos en el último tiempo en sus funciones sociales, culturales y políticas, proponiendo nuevas perspectivas, paradigmas y problemáticas en su abordaje, los cuales han impactado profundamente tanto en el proceso enseñanza - aprendizaje de los estudiantes, como en la labor y rol docente. Estos cambios deben ser contextualizados en una sociedad globalizada y mediada por fenómenos sociales complejos; el avance de la ciencia y, dentro de ellas, las telecomunicaciones; así como también la multiplicidad de alternativas educativas han generado un nuevo escenario para toda la comunidad. De esta manera, la educación a distancia, por sus características y por la potencialidad que ofrecen las tecnologías de información y comunicación que ahora tiene a su alcance, se convierte en una alternativa importante para lograr que la educación superior llegue a todos los sectores de la sociedad (Morresi \& Donnini, 2007).

El incremento de las modalidades de estudio no tradicionales, ya sea e-learning o blearning, son visibles y considerables en países europeos y asiáticos. Por su lado, países latinoamericanos como México y Costa Rica han considerado estas modalidades de estudios para dar cobertura e inclusión social a sus comunidades. Por su parte, el Ministerio de Educación de Chile, señala que del total de alumnos cursando una carrera de educación superior de pregrado en el año 2016, el 2,1\% lo realiza en un programa a distancia y $0,6 \%$ en semipresencial, porcentajes aun marginales comparados con la modalidad presencial.

En este sentido, la Universidad UNIACC ha sido pionera en el país respecto a su oferta de programas de pregrado en modalidades e-learning y b-learning, tales como las carreras de Ingeniería Comercial y Psicología, las cuales se constituyen como proyectos educacionales que han sido acreditados por la Comisión Nacional de Acreditación de Chile, mostrando de esta manera un sólido sello de calidad en su propuesta formativa.

Un desafío importante que tienen las universidades se refiere a que sus estudiantes permanezcan en las carreras y logren titularse en tiempos adecuados, por ello las instituciones de educación superior han establecido diferentes estrategias para potenciar la persistencia académica de los estudiantes y disminuir la deserción universitaria, fenómeno relevante de considerar dados los costos que genera tanto a las universidades, como a los estudiantes y al Estado. Por lo anteriormente expuesto, llama la atención que el fenómeno de la deserción universitaria tenga escasa investigación, situando sólo el tema en la magnitud del problema. González (2005, en Rubio, 2011) señala que la tasa de deserción a nivel de pregrado en el año 2004 era cercana al $53.7 \%$, siendo mayor en universidades privadas que en públicas, lo que 
significaría que desertaría del sistema de las universidades más de la mitad de los estudiantes que ingresan a ella. De modo más actualizado, el Sistema de Información de Educación Superior (SIES), informó el año 2017 que 1 de cada 3 estudiantes abandona la universidad en el primer año, fenómeno que ha aumentado en un 2,6\% desde el año 2011 al año 2015. De las escasas investigaciones que intentan conocer factores que se asocien a la deserción se puede concluir que uno de ellos y relevante de considerar es el acceso al financiamiento de la carrera, el cual reduciría las probabilidades de deserción.

Respecto a la deserción de los estudiantes que estudian en modalidad semipresencial en Chile no fue posible encontrar estudios o investigaciones que apunten a factores, causas o comprensiones del fenómeno, lo que da pie a interesarse por la temática, considerando que esta envuelve las esferas sociales, políticas, económicas vinculadas al desarrollo del país. Tal como fue señalado, dada la escasa literatura encontrada que diera cuenta, más allá de las cifras de deserción universitaria, se decidió explorar el fenómeno desde la visión de uno de los actores claves dentro de la comunidad educativa: los docentes.

\section{Objetivo General}

Explorar las representaciones que tienen docentes universitarios de la universidad UNIACC respecto al fenómeno de la deserción de los alumnos de primer año que cursan estudios en modalidad semipresencial.

\section{Objetivos Específicos}

Los objetivos específicos, que responden al objetivo general, son:

1. Conocer las ideas y creencias que manejan los docentes universitarios respecto del fenómeno de la deserción universitaria en los estudiantes de primer año de modalidad semipresencial.

2. Conocer el campo representacional de los docentes de primer año respecto a la deserción universitaria en la modalidad de estudios semipresencial.

\section{Metodología}

Para responder a la pregunta de investigación: “¿Cuáles son las representaciones de docentes universitarios respecto a la deserción de sus estudiantes de primer año en modalidad de estudios semipresencial en la universidad UNIACC?" se realizó un estudio cualitativo de corte transversal con alcance exploratorio. 
Tabla $\mathbf{N}^{\circ} 1$ Dimensiones abordadas en guion temático

\begin{tabular}{|r|l|l|}
\hline Número & \multicolumn{1}{|c|}{ Nombre de dimensión } & $\begin{array}{c}\text { Número de temas } \\
\text { por dimensión }\end{array}$ \\
\hline 1 & Percepción Inicial Intuitiva & 5 \\
\hline 2 & Relación profesor - alumno & 3 \\
\hline 3 & Características del estudiante & 1 \\
\hline 4 & Características del profesor & 1 \\
\hline 5 & Estrategias que utilizan los docentes & 3 \\
\hline 6 & Deserción en primer año & 3 \\
\hline
\end{tabular}

Fuente: Elaboración propia

La tabla $\mathrm{N}^{\circ} 1$ muestra las seis dimensiones que constituyeron para realizar el guion temático, cada dimensión incorporó un número de temas relacionados, los cuales dependiendo de cada dimensión, varían entre 1 a 5 temas. Con ello, se realizó un grupo focal, compuesto por cinco profesionales internos de la universidad que se desempeñan o se han desempeñado realizando docencia en programas académicos de la universidad, tanto presencial como semipresencial u online.

El grupo focal se realizó en sala espejo y estuvo guiado por el investigador responsable del proyecto, estando tras el espejo unidireccional las co investigadoras, quienes tuvieron la función de evaluar el focus tanto en términos de forma como de contenido.

Luego de la realización del grupo focal, en un ejercicio de reflexión, los investigadores decidieron acotar el objetivo del estudio hacia las representaciones docentes únicamente de los estudiantes semipresenciales, dejando de lado las representaciones de los estudiantes vespertinos, aspecto incorporado en el proyecto inicial. Se definió esta resolución dado que emergieron del Grupo Focal distinciones de relevante consideración entre las características del estudiante que realiza su proceso formativo en modalidad semipresencial, los atributos de los docentes que trabajan en dicha modalidad y el vínculo estudiante - universidad que se crea en la semipresencialidad, en contraste con los estudiantes presenciales.

Por otra parte, también se decidió realizar un cambio en la técnica de recolección de datos, definiendo que se realizarían entrevistas semiestructuradas, a partir del guion temático, para abordar más profundamente las representaciones de los docentes respecto a los estudiantes semipresenciales que abandonan la universidad durante el primer año. 


\section{Participantes}

El universo del estudio estuvo compuesto por cuatro docentes de la universidad UNIACC que han realizado asignaturas en las carreras de Psicología y Trabajo Social. La muestra fue no probabilística, de tipo voluntaria.

Se consideraron como criterios de inclusión, académicos de la Universidad UNIACC que realizaran docencia en el primer año de carreras semipresenciales pertenecientes a la Facultad de Humanidades y Ciencias Sociales, los cuales tuviesen una experiencia superior a un año en dicha actividad.

A continuación se presenta una caracterización de los docentes entrevistados.

Tabla $\mathbf{N}^{\circ} \mathbf{2}$ Caracterización de docentes entrevistados

\begin{tabular}{|c|l|c|c|}
\hline Nombre & Sexo & Edad & $\begin{array}{c}\text { Años de docencia } \\
\text { semipresencial }\end{array}$ \\
\hline E1 & Femenino & 38 & 5 \\
\hline E2 & Masculino & 64 & 2 \\
\hline E3 & Femenino & 39 & 2 \\
\hline E4 & Femenino & 63 & 5 \\
\hline
\end{tabular}

Fuente: Elaboración propia

La tabla $\mathrm{N}^{\circ} 2$ muestra que tres de los cuatro entrevistados son mujeres y las edades fluctúan entre 38 y 64 años. Aun cuando hay una heterogeneidad respecto a la edad, los años impartiendo docencia en modalidad semipresencial en la universidad UNIACC varían entre 2 a 5 años.

\section{Resultados}

Del análisis basado en la Teoría Fundamentada (Glaser \& Strauss, 1967), desarrollado en base a las entrevistas realizadas, emergieron categorías descriptivas y categorías relacionales. Respecto a las categorías descriptivas, producto de la codificación abierta, emergen cuatro temas que serán presentados a continuación, explicando sus características principales y presentando evidencia textual que los avala.

\section{1.- Categorías descriptivas}

\subsection{Características de los estudiantes}

Esta categoría hace referencia a la manera en que los docentes entrevistados describen las características de los estudiantes que estudian en modalidad semipresencial y se configura desde dos vértices; estudiantes que se mantienen o 
persisten y estudiantes que fracasan y terminan desertando en la carrera.

Respecto a los estudiantes que se mantienen en la carrera, los docentes entrevistados señalan que son alumnos orientados al logro académico, quienes tienen buenos rendimientos generales en término de obtener buenas evaluaciones tanto en las actividades online como en los exámenes presenciales de la carrera. Además son personas que presentan un interés genuino en la formación profesional, lo cual se observa en que logran involucrarse en las diferentes actividades académicas, son proactivos en buscar soluciones a las dificultades que enfrentan y buscan interacción con profesores y compañeros para responder a sus dudas y señalar sus puntos de vista.

En contraste, los estudiantes que fracasan y terminarían desertando del proyecto universitario, son aquellas personas que no se esfuerzan en su proceso de aprendizaje, lo que se vería reflejado en que no generan preguntas frente a sus inquietudes, demuestran poco interés en el estudio y no tendrían un interés genuino en aprender. Además, se releva que una parte de estos estudiantes tendrían dificultades cognitivas y/o de aprendizaje, los cuales tenderían a desertar rápidamente por el fracaso académico en las primeras asignaturas de la carrera, en contraste con estudiantes que pudiesen tener otro tipo de problemáticas; afectivas, de personalidad o anímicas que no serían impedimento para mantenerse en la universidad.

Tener expectativas no realistas también sería una característica de los estudiantes que fracasan, las cuales serían tanto hacia la carrera como a la modalidad de estudios semipresencial, develándose en este aspecto prejuicios asociados a que esta modalidad de estudios es rápida, fácil, no requiere dedicación y cualquier persona es capaz de hacerlo sin esfuerzo.

Por último, los docentes describen dificultades extra académicas que viven los estudiantes que afectarían su permanencia en la carrera; entre ellas se señalan dificultades laborales; tales como cambios de turnos laborales, cambios de empleo y despidos, dificultades económicas y otras dificultades personales no contenidas anteriormente, como problemas de pareja o dificultades con sus hijos, por ejemplo.

\section{2.- Construcción de la relación profesor - estudiante}

Esta categoría emerge de las representaciones de los docentes entrevistados, considerando la centralidad de la construcción de la relación profesor - estudiante a través de la co - construcción de esta relación, en donde se visualiza una relación simétrica y bidireccional, en el sentido de que se describe un proceso en donde el 
estudiante aprende del docente, como también el docente aprende del estudiante, explicitando que el primero no es un sabio o conocedor de toda la verdad. Se lograría esta relación a través de los espacios didácticos disponibles; tales como foros de clases, correos electrónicos y avisos del docente en plataforma online, como también a través de las clases presenciales en donde se permite la consolidación de la relación. La construcción de la misma también involucra atender oportunamente a las necesidades de los estudiantes, percibiendo sus necesidades tanto desde el entorno virtual de aprendizaje como desde las clases presenciales. Cabe señalar que se devela que esta construcción también conlleva la creación de espacios de confianza por parte del docente y requiere un esfuerzo, por ejemplo, para motivar al estudiante y conocer a la persona tras el computador.

Por último, la construcción de la relación profesor - estudiante le daría sentido al proceso formativo, convirtiéndose para ambos actores en una experiencia educativa.

\subsection{Uso de estrategias docentes}

La presente categoría da cuenta de la manera en que el uso de estrategias docentes tales como invitar a reflexionar y preguntar por las dificultades en la comprensión de los contenidos tratados por los docentes para obtener retroalimentación del aprendizaje del estudiante puede ser decisivo en la permanencia o deserción universitaria. Los docentes entrevistados relevan que usar estrategias, como ofrecer ayuda y apoyo, por ejemplo, a través de andamiaje y utilización de recursos institucionales (Programa de Atención Psicoeducativo) sería beneficioso para la permanencia de los estudiantes. Lo anterior también tendría que ver con características personales del docente, ya que, de acuerdo estas, se utilizarían o no estrategias docentes y se seleccionarían cuáles estrategias utilizar. Se destacan las variables afectivas del docente, tales como el respeto, acogida y calidez como aspectos que contribuirían a prestar atención y preocupación por los estudiantes.

Por otra parte, ignorar a los estudiantes o no utilizar estrategias docentes pertinentes, tales como no entregar retroalimentación en tiempo y forma, son reconocidos como aspectos que dificultarían la permanencia en la universidad.

\subsection{Recomendaciones para prevenir la deserción}

La presente categoría dice relación con una serie de recomendaciones que reconocen los docentes para prevenir la deserción universitaria; considerando que se parte de la base de que no es posible garantizar la permanencia de todo el alumnado.

Los docentes entrevistados mencionan que sería importante diferenciar aún más al 
estudiante semipresencial del presencial para reconocer las características específicas de los primeros e intervenir con ellos de manera más precisa. Así mismo, señalan que la universidad debiese fijar un discurso de flexibilidad con límites frente a las demandas de los estudiantes, tanto en términos académicos como administrativos, lo que conlleva una implementación de estrategias institucionales para abordar específicamente las dificultades de aprendizaje de los estudiantes de manera personalizada, es decir, operar preventivamente más que reactivamente frente a este fenómeno, detectando tempranamente a estudiantes con estas dificultades y abordándolas, por ejemplo, con un programa de re-educación de hábitos de estudio.

También, los docentes señalan que sería importante centrarse en hacer obligatorias ciertas metodologías con recursos que ya existen en la universidad, se plantea la idea de generar encuentros virtuales antes de las clases presenciales como medida anticipatoria que favorezca la motivación en los estudiantes.

Además, los docentes entrevistados señalan que sería importante fomentar aún más la relación de los docentes con sus escuelas, para tener un mecanismo de entrega de información a la unidad de estudiantes que no están participando de manera online, lo que permitiría generar sistemas de acompañamiento y seguimiento más efectivos.

Dado el alcance de la investigación, las cuatro categorías emergentes dan cumplimiento al objetivo de la investigación, no obstante se permite indagar de manera más compleja los fenómenos que emergen del análisis más profundo, exponiendo relaciones entre las categorías, a continuación.

\section{2.- Categorías relacionales}

A continuación se presentan dos categorías relacionales a través de las que se favorece la comprensión del fenómeno de la deserción universitaria, desde la perspectiva de los docentes entrevistados. 


\section{1.- Fenómeno: Desmotivación}

Figura 1: Fenómeno “Desmotivación”

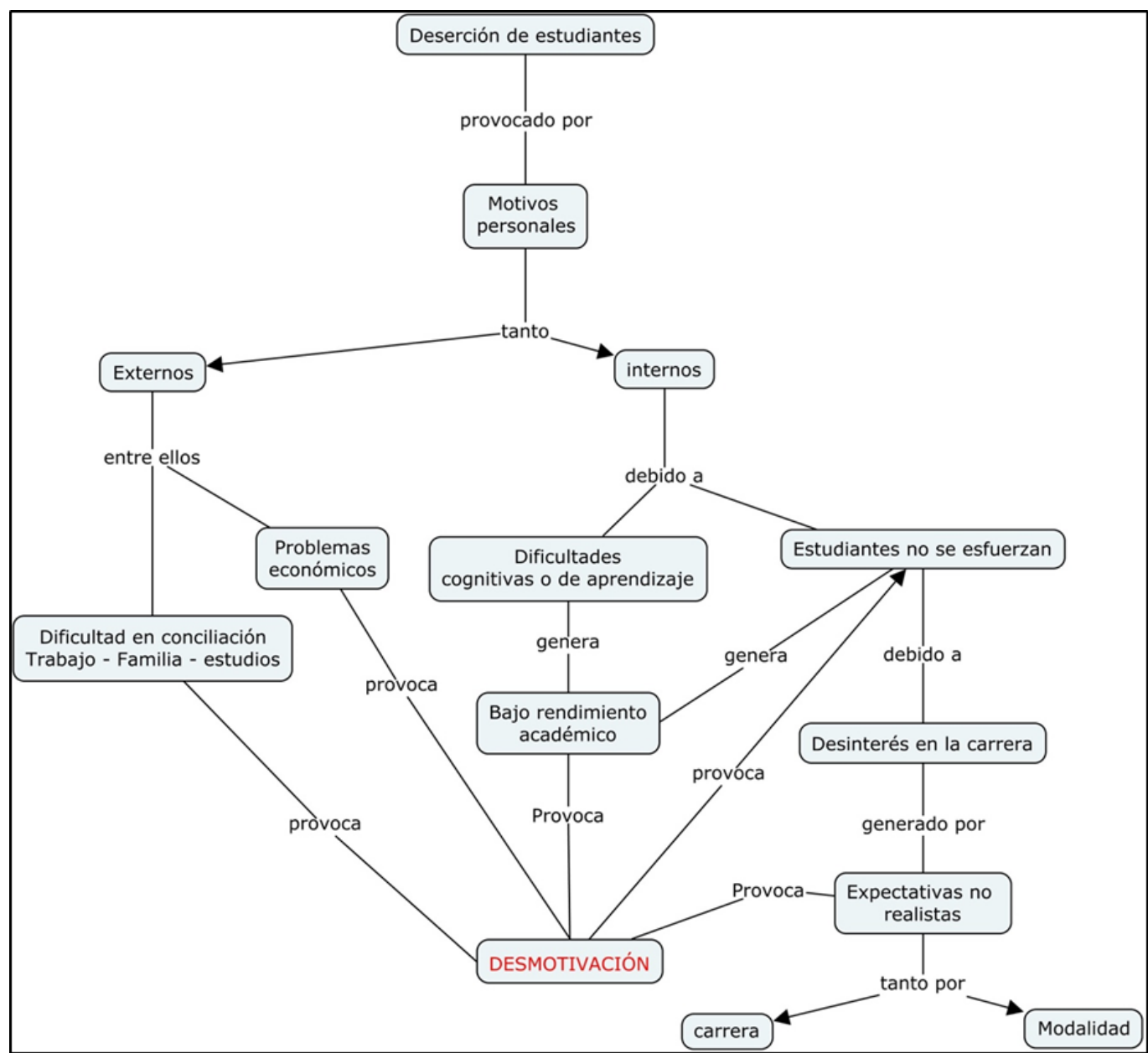

Fuente: Elaboración propia

La figura $\mathrm{N}^{\circ} 1$ permite observar la relación que establecen los docentes entrevistados como causas de la deserción de los estudiantes de primer año en modalidad de estudios semipresencial de la universidad UNIACC.

La desmotivación del estudiante aparece como un eje axial frente al fenómeno de deserción universitaria, la cual es percibida como el efecto tanto de aspectos externos como internos del propio estudiante.

Los entrevistados destacan como aspecto externo que desmotiva a los estudiantes, respecto de continuar la carrera universitaria que han elegido, la dificultad que tienen éstos para compatibilizar de manera efectiva trabajo, familia y estudios en términos de tiempo y dedicación a estas tres esferas de la vida. Existirían problemas laborales que le impedirían al estudiante cumplir con las obligaciones que requiere la modalidad semipresencial, como por ejemplo, asistir a clases presenciales debido a 
turnos de trabajo los fines de semana en que se requiere la presencialidad en la universidad. Por su parte, la familia jugaría un factor importante ya que gran parte de los estudiantes serían mujeres quienes, a su vez, son identificadas como responsables del cuidado de los hijos o parientes enfermos, actividades que tendrían que conciliar con el estudio, lo que impediría responder adecuadamente a los tiempos de dedicación que requiere la carrera, haciéndose presente de esta manera una dificultad asociada a los roles de género culturalmente construidos.

Los problemas económicos son relevados como explicación de la desmotivación de los estudiantes, en tanto estos no pueden cumplir con las exigencias de la carrera; por ejemplo, se considera relevante plantear el costo que deben asumir las familias de los estudiantes que viven en regiones fuera del área metropolitana, respecto a gastos asociados a la asistencia a clases presenciales.

Dentro de los factores internos que intervienen en la desmotivación, emergen de las entrevistas las dificultades cognitivas o de aprendizaje de los estudiantes, las cuales provocarían un bajo rendimiento académico, el que a su vez fomentaría la desmotivación por continuar estudiando. El bajo rendimiento, a su vez, es percibido como causa del bajo esfuerzo de los estudiantes en su proceso de aprendizaje y la desmotivación por el bajo rendimiento, reforzaría que los estudiantes pierdan interés y ganas de esforzarse en el contexto de su proceso formativo.

Otro aspecto relevante vinculado el escaso esfuerzo del estudiante respecto a su proceso de aprendizaje, sería la falta de interés en la carrera, lo cual estaría relacionado con las expectativas no realistas, en relación a la carrera y modalidad de estudios. A su vez, las expectativas no realistas mantendrían la desmotivación que pueden sentir los estudiantes.

En síntesis, las causas de la desmotivación de los estudiantes tienen que ver con la dificultad de conciliar trabajo - familia y estudios, problemas económicos, el bajo rendimiento académico y expectativas no realistas de la carrera y de la modalidad. La consecuencia de la desmotivación provocaría que los estudiantes no se esfuercen para lograr buenos resultados académicos, perdiendo interés en la carrera. 


\section{2.- Fenómeno: Frustración y malestar del estudiante}

Figura 2: Fenómeno Frustración y malestar del estudiante

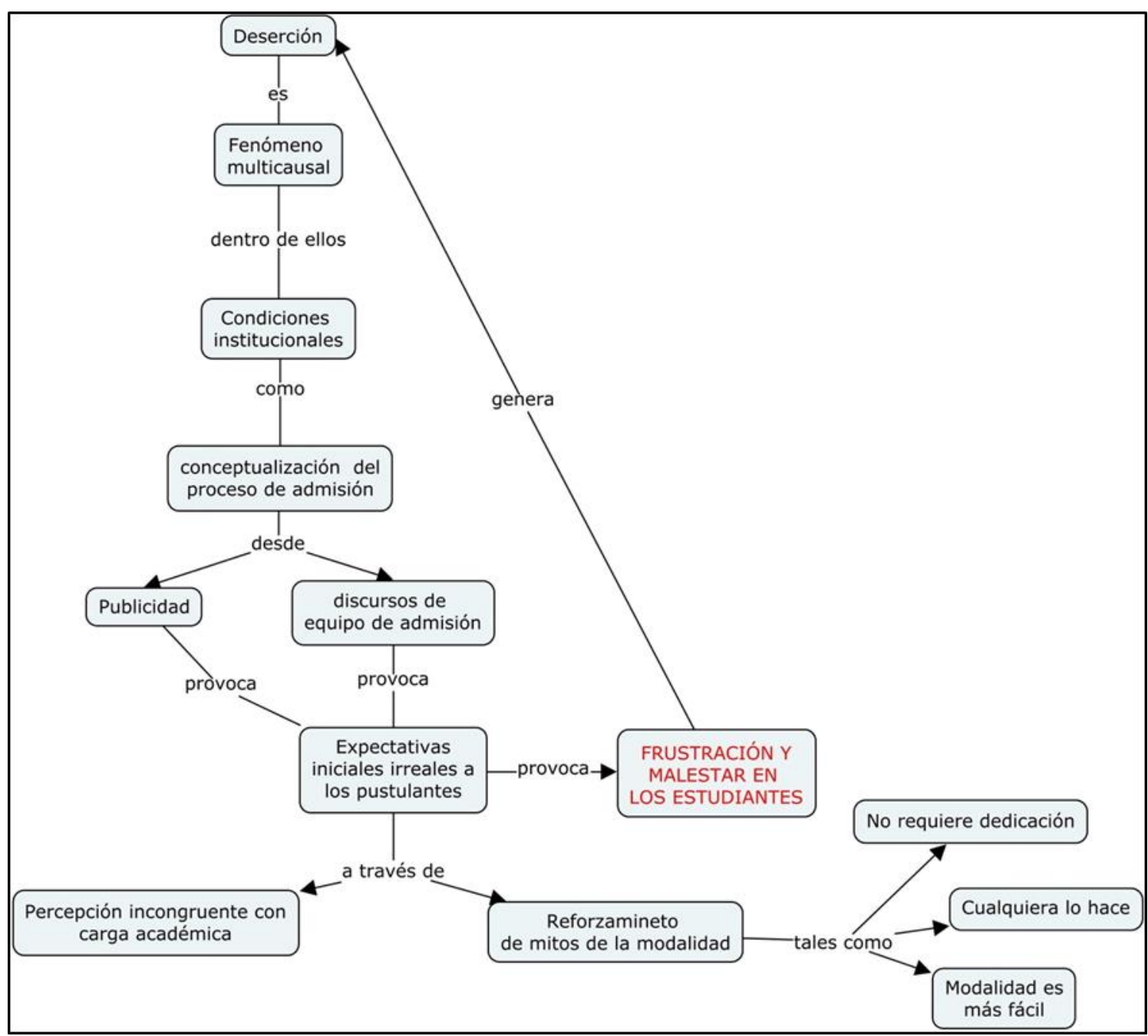

Fuente: Elaboración propia

La figura $\mathrm{N}^{\circ} 2$ permite observar las relaciones que se establecen para comprender la frustración y malestar de los estudiantes de la modalidad semipresencial, el cual es el centro del presente eje axial. Aun cuando los docentes reconocen que la deserción es un fenómeno multicausal, uno de los factores que emergen de las entrevistas son las condiciones institucionales propias de la universidad, específicamente se alude a cómo la institución conceptualiza el proceso de matrículas, tanto desde la publicidad que se trasmite al público como por el discurso de admisión, ambos elementos generarían expectativas iniciales irreales a los postulantes respecto a la modalidad de estudios semipresencial. Estas expectativas irreales son contrarrestadas, posteriormente, por la carga académica real que exigen los programas de pregrado, lo que generaría una percepción de incongruencia, que a su vez, se devela que las expectativas irreales refuerzan mitos asociados a la modalidad de estudios 
semipresencial, relacionados con que no requiere dedicación y/o que tiene menor nivel de dificultad.

Por lo anteriormente expuesto, se identifica como causa de la frustración y el malestar de los estudiantes, las expectativas irrealistas con las cuales ingresan a las carreras y, que una vez dentro, se contrastarían con la experiencia de mayor exigencia y tiempo que el que planificaron inicialmente. Como consecuencia, el estado anímico de malestar y frustración generaría una evaluación y finalmente la decisión de desertar del proceso formativo.

\section{Discusión y conclusiones}

La Educación Superior ha experimentado grandes cambios en los últimos 20 años, tanto en sus dimensiones sociales, como culturales y políticas. Tal como señala Salmi (2013), las telecomunicaciones y la globalización han creado un sinfín de oportunidades que la educación ha tomado para crear y transformar la manera de conceptualizar el proceso de enseñanza. Chile no ha estado ausente de estos cambios globales en Educación Superior, incorporando lenta y paulatinamente la educación a distancia como una manera de brindar oportunidades de formación universitaria a todas las personas que, por distintos motivos, no pueden realizar un proceso formativo universitario de manera presencial, orientándose por ejemplo a personas que trabajan.

Por su parte, la educación a distancia, ya sea en formato semipresencial (b- learning) o virtual (e-learning) tiene características similares y otras diferenciadoras si se compara con la educación presencial tradicional. Entre las características comunes se pueden señalar: contar con un marco regulatorio político que resguarda la calidad de la educación, modelos epistemológicos, paradigmáticos y teorías curriculares que sustentan los programas de estudios, modelos educativos institucionales que definen y delinean el proceso formativo, los perfiles de egreso y la gestión académica de los estudiantes, participantes y roles comunes en el proceso, tales como docente y estudiante, planificaciones que guían el proceso de aprendizaje, entre otras. Las diferencias tienen que ver, principalmente, con las metodologías y didácticas con las cuales se lleva a cabo el proceso de aprendizaje, el uso de Entornos Virtuales de Aprendizaje (EVA) y mecanismos evaluativos propios de cada modalidad.

De esta manera, independientes a la modalidad de estudios, surgen fenómenos comunes en Educación Superior; uno de ellos es la deserción universitaria, aspecto relevante que dice relación con el hecho mediante el cual un estudiante interrumpe sus estudios, sin haber cubierto en su totalidad el plan de estudios de la carrera 
respectiva, y que ha sido abordado en esta investigación.

De acuerdo a Díaz - Peralta (2008), existen diferentes motivos por los cuales las personas desertan en la Educación Superior. De forma coherente con los hallazgos encontrados en la presente investigación, éstos se vinculan principalmente con elementos asociados a aspectos socioeconómicos (pérdida de empleo, problemas familiares, multiplicidad de funciones), individuales (desmotivación, falta de interés por la carrera, ausencia de esfuerzo, expectativas poco realistas en relación a la modalidad), institucionales (tensión entre: la difusión de la modalidad y las exigencias académicas para pregrado, delimitación del concepto de "flexibilidad") y académicas (baja autonomía docente en la modalidad).

Bean y Metzner (1985, en Canales \& De los Ríos, 2018) destacan la importancia de las variables de contexto social y económico de los alumnos como causas de deserción. Entre los factores más relevantes consideran la situación financiera del estudiante y su familia, trabajo (horas de empleo) y las responsabilidades familiares (p.177). Lo anterior es concordante con los resultados obtenidos en la primera codificación axial, en donde los docentes manifiestan que las tensiones de conciliar estudios familia y trabajo afectan la motivación de los estudiantes, por lo que se concluye que existente coherencia entre el hallazgo de la investigación con lo indicado en la literatura, siendo un aspecto que implicaría desmotivación en los estudiantes. En este sentido, se destaca la relevancia de potenciar estrategias institucionales que permitan a los estudiantes organizar o planificar los tiempos relativos al estudio, considerando los diferentes roles que los propios estudiantes ejercen en los diferentes ámbitos de la vida. Además, se destaca el valor de fomentar el reconocimiento de las potencialidades para hacerse cargo de los desafíos académicos, a través de una comunicación clara, que favorezca la construcción de expectativas realistas respecto al proceso de formación.

Por su parte, los docentes entrevistados señalan que el bajo rendimiento académico sería un factor que generaría desmotivación en los estudiantes. En este sentido, las variables cognitivas de los estudiantes serían uno de los principales factores predisponentes para un bajo rendimiento, no obstante la literatura indica que si bien pueden ser considerados como predictores, existen otros aspectos que influyen en este fenómeno (García, Y., López de Castro, D., \& Rivero, O. 2014). Frente a lo anterior, es importante poder deconstruir estereotipos que los docentes pueden tener de los estudiantes con bajo rendimiento académico; en este sentido, dichos estudiantes, generalmente tienen dos dificultades primordiales: no saben estudiar y no saben aprender (Abarca y Sánchez, 2010 en García, Y., López de Castro, D., \& Rivero, O. 2014). Por lo general, los estudiantes con bajo rendimiento académico 
tienen una percepción negativa de sí mismos (Rodríguez, 2016) y asumen que su bajo rendimiento se explica por sus deficiencias cognitivas, idea que podría ser reforzada por los mismos docentes, a través de la comunicación de expectativas acerca del rendimiento. Por lo anterior, es importante poder intervenir con los docentes frente a las creencias que pueden tener de este "tipo" de estudiantes, propiciando una visión más integral respecto de los factores que influyen en el bajo rendimiento académico de los estudiantes, como por ejemplo problemas emocionales, afectivos y tensiones internas debido a múltiples causas y circunstancias personales y ambientales.

En este sentido, cobra relevancia que el docente resignifique su rol en el contexto de esta modalidad, puesto que si bien se describen una serie de estrategias asociadas a elevar los niveles de motivación de los estudiantes, generación y establecimiento de vínculos, relevando la significancia de estos y el abordaje personalizado de los estudiantes que presentan ausentismo en foro y plataforma en general, no es posible observar en sus discursos elementos o estrategias concretas asociadas al abordaje que realizan con los estudiantes que evidencian bajo rendimiento académico. En este sentido, se destaca la necesidad de que los docentes tengan formación en el ámbito educacional, para abrir posibilidades de abordaje mejor estructuradas y metodológicamente sustentadas en la evidencia; esta necesidad ha sido comunicada por autores como Llerena (2015). Complementando aquello, y en función de las formas descritas por los docentes para prestar apoyo a distancia a un estudiante con mal desempeño académico, Silva (2011) identifica la relevancia de poder dar información a los docentes de los medios que cuenta el ambiente virtual de aprendizaje más allá del correo, como por ejemplo, cafés virtuales, así como fomentar el uso efectivo de estas herramientas pedagógicas, en función del logro de aprendizajes conceptuales, procedimentales y actitudinales. En este sentido, la universidad, con la oferta formativa dirigida a los docentes de todas las carreras hace un reconocimiento de esta necesidad y la aborda.

Respecto de las expectativas, Ulloa (2017) coincide con los hallazgos de esta investigación, en la que los entrevistados comparten su visión al respecto de que la retención se ve interferida por el conocimiento que tienen los estudiantes del proyecto educativo en que están depositando su confianza, así como de la modalidad de estudios. Por eso se hace necesario que la información que se transmite en el momento de la matrícula sea realista y objetiva, de modo que los estudiantes que ingresen tengan una representación lo más apegada a la realidad posible, acerca de las posibilidades que se les ofrecen así como de las potencialidades que deberán desarrollar para persistir en este proyecto académico. 


\section{Limitaciones y recomendaciones}

Dadas las dificultades que se presentaron para convocar a los participantes, se tomó la decisión de sustituir el instrumento de recolección de datos inicialmente elegido (grupo focal) por la entrevista semiestructurada, cuya pauta fue elaborada en función de los objetivos de investigación, a la vez que tomando los elementos relevantes del grupo focal realizado inicialmente (como pilotaje). Este cambio en el diseño metodológico podría haber limitado los resultados, considerando que la entrevista no ofrece la oportunidad de surgimiento de nuevas ideas a raíz de la interacción entre los participantes (como sí lo posibilita el Grupo Focal); sin embargo, fue posible profundizar en algunas opiniones, facilitando también la sinceridad de los participantes, ante la privacidad que ofrecía este instrumento (Flick, 2012).

En relación con lo anterior, se encontraron dificultades para lograr un número mayor de participantes, lo que pudiera haber enriquecido los resultados, sin embargo, las coincidencias en los contenidos que emergieron en las respuestas de los docentes entrevistados, indican que estos participantes no sólo se están refiriendo a su experiencia individual, sino a las representaciones construidas a través de vivencias compartidas y comunicadas por otros colegas y por sus estudiantes -cuestión que quedó explícita en las entrevistas- y que coinciden con los relatos de los alumnos a los que estos investigadores también han podido acceder en el contacto cotidiano. Esto es coincidente con lo propuesto por Rodríguez (2006), quien afirma que "las representaciones son necesariamente la expresión de algún grupo" (p. 85).

Pese a las limitaciones mencionadas, esta investigación ofrece luces no sólo sobre los resultados que ya fueron expuestos, sino además sobre temáticas que se consideran relevantes para futuros estudios. Es así que se sugiere indagar en las representaciones de los estudiantes vigentes respecto a las condiciones que favorecen su permanencia; así como en las de aquellos que han decidido interrumpir sus estudios, indagando en el proceso de toma de decisiones: recursos de apoyo, razones que los llevaron a tomar esta determinación, atribuciones causales de la deserción, etc., para apuntar al logro de una teoría fundamentada en aquellos fenómenos que fueron identificados a través de la codificación axial.

Finalmente, considerando los resultados obtenidos en esta investigación, recomendamos fomentar una relación más cercana y cotidiana entre las unidades académicas y otras áreas de la universidad para favorecer el conocimiento mutuo y el trabajo conjunto, a modo de evitar la generación de expectativas irreales en los postulantes, que pudieran verse refutadas al ingresar a las carreras. 


\section{Referencias}

Canales, A., \& De los Ríos, D. (2018). Factores explicativos de la deserción universitaria. Calidad en la Educación, (26), 173-201. doi: 10.31619/caledu.n26.239

Díaz-Peralta, C. (2008). Modelo conceptual para la deserción estudiantil universitaria chilena. Estudios Pedagógicos, 34(2), 65-86.

Flick, U. (2012). Introducción a la investigación cualitativa (3a. ed.). Recuperado de https://ebookcentral.proquest.com/lib/bibliouniaccsp/reader.action?ppg=130\&do clD=3217765\&tm=1544202885111

García, Y., López de Castro, D., \& Rivero, O. (2014). Estudiantes universitarios con bajo rendimiento académico, ¿Qué hacer?. Edumecentro, 6(2), 272-278.

Glaser, B. y Strauss, A. (1967). The Discovery of Grounded Theory: Strategies for qualitative research. New York, NY: Aldine.

Llerena, C. (2015). Modelo de orientación profesional para el desarrollo de la motivación hacia la profesión en estudiantes de la modalidad semipresencial de carreras sociohumanísticas. Tesis para optar al grado científico de Doctor en Pedagogía. Universidad de Ciencias Pedagógicas "Manuel Ascunce Domenech", Cuba. Recuperado de https://ebookcentral.proquest.com/lib/bibliouniaccsp/reader. action?ppg=3\&docID $=4183751 \& \mathrm{tm}=1544206688405$

Morresi, S., \& Donnini, N. (2007). Modalidad de educación semipresencial. Relato de una experiencia. Recuperado de http://repositorio.ufsc.br/xmlui/handle/ $123456789 / 82843$

Rubio, A. B. (2011). Deserción universitaria en Chile: incidencia del financiamiento y otros factores asociados. Revista CIS, 9(14), 59-72.

Rodríguez, C. (2006). Las representaciones sociales: entretejidos de la razón y la cultura. Recuperado de https://ebookcentral.proquest.com/lib/bibliouniaccsp/reader. action?ppg=2\&docID=3171807\&tm=1544204893581

Rodríguez Portuguez, G. (2016). Funciones ejecutivas, rasgos de personalidad y rendimiento académico en estudiantes universitarios de ciencias de la salud (Vol. 398). Ediciones Universidad de Salamanca. Recuperado de http://ebookcentral.proquest.com/lib/bibliouniaccsp/detail.action?doclD=490895 5. 
Salmi, J. (2013). Video Conferencia Magistral "Nuevos Desafíos para la Educación Superior en el siglo XXI", Universidad de Guadalajara, México.

Silva, J. (2011). Diseño y moderación de entornos virtuales de aprendizaje (eva). Recuperado de http://ebookcentral.proquest.com/lib/bibliouniaccsp/detail. action?docID=3201623

Ulloa, M. (2017). Análisis sobre la deserción de alumnos: abocado a su retención, en una universidad privada. Recuperado de http://ebookcentral.proquest.com/lib/bibliouniaccsp/detail.action?doclD $=548608$ $\underline{0}$

\section{Cristian Ortega Bustos}

Psicólogo, Universidad de Concepción. Magíster en Docencia Universitaria, Universidad del Desarrollo, Postítulo en Psicoterapia Focal Psicoanalítica, Corporación Salvador - Universidad de Chile. Psicólogo Acreditado como Especialista en Psicoterapia, Comisión Nacional de Acreditación de Psicólogos Clínicos, Docente y Director Escuela de Psicología Universidad UNIACC.

\section{Anais Aluicio González}

Psicóloga, Universidad Santo Tomás. Magíster en Educación y Formación Universitaria, Universidad San Sebastián. Postítulo en Psicoterapia Cognitiva Postracionalista, Sociedad de Psicoterapia Cognitiva Postracionalista. Docente y Coordinadora Programa de Atención Psicoeducativa, Universidad UNIACC.

\section{Edith Jorquera Muñoz}

Asistente Social y Licenciada en Trabajo Social, Universidad de Los Lagos. Magíster en Planificación y Gestión de Políticas Públicas, Universidad Central. Diplomado en Gerontología Social, Pontificia Universidad Católica de Chile. Docente y Directora Escuela de Humanidades, Universidad UNIACC. 